Actes du 16e colloque du GERAS

\title{
ESP : l'ouverture sur un monde en mutation
}

\section{Evelyne Brouzeng}

\section{OpenEdition}

\section{Journals}

Édition électronique

URL : http://journals.openedition.org/asp/3933

DOI : $10.4000 /$ asp.3933

ISSN : 2108-6354

\section{Éditeur}

Groupe d'étude et de recherche en anglais de spécialité

\section{Édition imprimée}

Date de publication : 1 décembre 1995

Pagination : 303-310

ISSN : 1246-8185

\section{Référence électronique}

Evelyne Brouzeng, «ESP : I'ouverture sur un monde en mutation », ASp [En ligne], 7-10 | 1995, mis en ligne le 01 novembre 2013, consulté le 20 avril 2019. URL : http://journals.openedition.org/asp/3933 DOI : 10.4000/asp.3933

Ce document a été généré automatiquement le 20 avril 2019.

Tous droits réservés 


\title{
ESP : l'ouverture sur un monde en mutation
}

\author{
Evelyne Brouzeng
}

1 En 1983, Widdowson dans son ouvrage Learning purpose and language use montrait comment la formation à l'ESP favorisait la définition d'objectifs précis et le développement de compétence spécifiques. Il prévoyait déjà qu'il s'agissait là d'un passage obligé, d'une étape sur un itinéraire qui débordait ce cadre bien défini des besoins particuliers et Widdowson disait alors :

The purposes in ESP are arranged along a scale of specificity with training at one

end and education at the other. $(1983: 10)$

2 Nous trouvons riche de promesses l'idée que la formation et l'éducation participent du même processus, impliquant par là que la formation sans la dimension éducation est à terme une démarche stérilisante. D’Espagnat (1990) reprenant le thème du danger de la « science sans conscience » nous invite à « penser la science » c'est-à-dire à accompagner l'enseignement des sciences d'une réflexion critique.

3 Pourtant, nous sommes réticents devant la représentation donnée par Widdowson d'une situation d'écartèlement entre deux pôles, la formation et l'éducation, comme si l'on formait d'abord pour éduquer ultérieurement. La poursuite d'objectifs de formation "utiles» et «immédiatement exploitables» nous ont parfois conduits à voir dans l'apprentissage de la langue 2 l'acquisition d'un outil; cette démarche fonctionnelle s'opposait par là, au nom de l'efficacité à toute prétention culturelle. Chacun d'entre nous n'a-t-il pas un jour entendu un commanditaire de formation l'exhorter : « Et surtout vous n'êtes pas là pour leur faire du Shakespeare !»?

\section{Formation et éducation}

4 L'idée d'une césure entre formation et éducation mérite d'être mise en débat. Au niveau individuel de l'apprenant et de l'enseignant, apprendre et enseigner induisent pour chacun des "acteurs» des enjeux identitaires et culturels. Johnson dans son ouvrage, Communication in the class-room (1981) y insiste ainsi : 
A consistent methodology is more than just a collection of activities and

techniques. It requires an underlying set of principles. (1981:59)

Pour préciser cet apport identitaire et culturel qui accompagne l'apprenant et l'enseignant dans leur parcours professionnel, il suffit d'évoquer la rencontre des collègues linguistes avec ce monde, qui, au départ, leur était totalement étranger: le monde des sciences et des techniques. Beaucoup furent au départ très effrayés de devoir se réduire à "traduire des notices techniques». Tous ont vite pris conscience que l'anglais pour des besoins spécifiques ne pouvait se réduire à cette activité parfois nécessaire mais jamais suffisante.

\section{Les métamorphoses de nos sociétés et leurs effets sur la formation}

6 Il n'y a pas si longtemps (dans les années 60) on considérait que la science et la technique se développaient de façon indépendante et que l'histoire de la technologie représentait celle du progrès. Cette histoire se divisait en étapes successives, commençant par la mécanisation (au XIX siècle), qui était suivie de l'automatisation (informatisation à la fin de la 2e guerre mondiale, machines à commande numérique dans les années 1970), et qui aboutissait à la robotisation, sans que soit fait systématiquement, dans la formation des techniciens, le rapprochement avec l'environnement économique, social et culturel qui génère ces mutations, et sans que soient envisagés les effets induits sur les hommes et le travail. Cette approche maintenait la technique à l'écart des préoccupations des citoyens et réduisait l'enseignement de l'ESP à une fonction instrumentale (comprendre la machine sans se préoccuper de la relation homme/machine et/ou machine/société).

7 Au début des années 80 , avec les premiers résultats du programme Fast (programme de recherche sur les changements scientifiques et techniques à long terme, sur leurs mécanismes, leurs implications et leurs conséquences pour les sociétés européennes) ${ }^{1}$ nous avons conscience de la complexité grandissante des relations entre développement sociétal, croissance économique, changements technologiques, emploi, travail. La crise de l'emploi et du travail a pris une dimension nationale et internationale.

8 Nous avons dû reconnaître que notre réflexion était rendue encore plus difficile par le fait que les situations étaient très diversifiées en fonction des branches d'industrie, des régions, des sexes et de l'âge. Les mutations de nos sociétés industrielles se traduisent aussi par l'évolution très rapide de facteurs de type qualificatifs comme les valeurs de nos sociétés. L'engouement pour des modèles de gagneurs, et la réévaluation des aspirations concernant le travail ont fait par exemple que le montant du salaire prime désormais sur le contenu du travail. Les attitudes des individus ont aussi changé vis-à-vis du travail intérimaire et la mobilité. Enfin, des modifications structurelles profondes ont affecté nos régions et redessiné les cartes de l'emploi (déclin des grands donneurs d'ordre de l'armement par ex. et disparition de la sous-traitance). Ces bouleversements n'ont pas été sans effets sur l'enseignement en général et l'ESP en particulier. 


\section{Croissance des incertitudes et remise en question de l'appareil de formation}

Face à la situation que nous venons de décrire, le système éducatif dans son ensemble se trouve interpellé et l'ESP n'échappe pas à cette remise en question. Autant il était facile de fixer des objectifs d'apprentissage en L2 quand on connaissait avec précision le contenu du travail d'un technicien supérieur d'électronique, autant cela se complique si celui-ci soupçonne qu'il risque de se retrouver bientôt au chômage. Le déclin des certitudes de jadis et la fragilité des informations concernant l'avenir, loin de nous rendre frileux et repliés sur îlots protégés, doivent nous encourager à nous ouvrir résolument sur des pistes à exploiter. L'un des effets positifs des avancées technologiques est de nous avoir fait passer brutalement d'une situation où nous étions pauvres en informations reçues en langue étrangère à une situation où chacun dispose de plus d'informations qu'il ne peut en consommer.

\section{L'ESP et son histoire passée et à venir}

10 À partir du moment où l'enseignement d'une langue est devenu partie intégrante des cursus scientifiques, le concept d'ESP a émergé et s'est petit à petit enrichi et structuré. Aujourd'hui nous avons plusieurs décennies de recul et possédons désormais une grande variété de matériaux d'enseignement et de recherche (ouvrages de didactique, comptes rendus d'expérience, méthodes, etc.) sans compter les livres de classe destinés aux apprenants. Le moment est peut-être venu de faire le point sur le chemin parcouru, de regrouper tous ces petits pas que constitue chacune des questions que nous nous sommes posées et de chercher dans l'éclectisme apparent de nos démarches la cohérence interne, pour reprendre une formule que Michel Perrin (1982) lançait il y a déjà près de quinze ans. Nous ne prétendons pas donner une réponse définitive à la question : quel ESP pour le troisième millénaire?

11 En revanche, nous nous efforcerons d'apporter un éclairage original et personnel sur des préoccupations que l'évolution de nos sociétés a fait apparaître. Notre réflexion s'est nourrie du travail réalisé au sein de notre équipe d'enseignantes d'ESP à des techniciens supérieurs d'électronique.

\section{L'ESP : un système ouvert sur nos sociétés}

12 Si nous devions décrire dans sa globalité le champ de l'ESP, il nous faudrait certainement recourir aux techniques multidimensionnelles, car notre discipline s'articule et se construit à partir d'éléments pluriels. L'ESP élabore sa problématique autour d'une double démarche : enseigner/apprendre, autour des apprenants, en tenant compte de données aussi contradictoires que la diversité de ces derniers, de leur unicité, mais aussi de leur profil globalisé.

13 Le matériel pédagogique destiné à la classe sera donc construit à partir du noyau dur qu'est la science et la technique, autour du «faciliteur » ou "médiateur » qu'est l'enseignant seul avec ses apprenants, enrichi par l'apport de l'équipe pédagogique, nourri par l'extérieur (l'environnement social et culturel). 
C'est ce dernier point, la définition des enjeux et des perspectives de l'ESP dans sa relation avec la société et la culture d'aujourd'hui, que le présent texte a pour objet de développer.

La possibilité qui nous est désormais offerte de modifier les pratiques d'acquisition et de gommer la frontière classe/loisir s'accompagne de l'inversion du lieu d'immersion en langue (la maison et non la classe). L'idée développée plus haut d'une échelle de temps (ou d'un ordre d'urgence) qui place la formation avant l'éducation est d'autant moins acceptable, que le développement personnel de chacun des apprenants s'inscrit (ou peut s'inscrire) désormais dans la continuité, école/« école parallèle» (télévision-radio)/ entreprise. À l'éducation formelle vient s'articuler l'éducation informelle.

\section{Vers des sociétés interculturelles}

6 Aujourd'hui la télévision nous propose un contact régulier avec la langue et la culture des autres. L'accès à ces informations est facilité par des techniques diversifiées, soustitrages, commentaires d'images, doublage, etc. Pour l'apprentissage des langues, la situation la plus souple est le sous-titrage, c'est-à-dire la mise en relation d'un texte simplifié (en L1) qui correspond à une traduction globalisante de la parole (entendue en L2) avec une image. Cette avancée dans l'appropriation par tous de moyens (gratuits) qui vous mettent en relation avec le monde est riche de potentialités : elle nous permettra de mieux comprendre les transformations que vivent nos sociétés.

«On ne peut connaître sa propre culture si l'on n'en connaît pas d'autres ", disait Linton il y a 200 ans. À ce jour, on évalue mal l'impact de cette situation de possible diversité culturelle. Quand on interroge nos étudiants, on s'aperçoit qu'un petit nombre seulement regarde les émissions comme l'Eurojournal ou le journal de CBS. On connait encore moins comment cette pratique de la connaissance de la culture des autres déterminera de nouveaux comportements en matière de communication. Comme ledit Fritz Wittek :

Nous ne savons pas et par conséquent nous avons du mal à imaginer comment l'éducation des enfants européens peut-être améliorée par le recours à ces situations de diversité culturelle.

Nous avons pu constater que, souvent, les difficultés langagières en L2 allaient de pair avec des difficultés en L1 et correspondaient à des paramètres individuels portant sur l'origine sociale. Le recours à une éducation interculturelle peut être le moyen de combler ces handicaps du repli sur des univers étriqués. Pourtant, si nous pensons que l'école doit utiliser ces substrats pédagogiques, cela nous place devant de nouvelles responsabilités. Il ne s'agit pas seulement de faire acquérir des compétences langagières en utilisant des outils disponibles, des images et des commentaires; il s'agit de favoriser le développement d'une dimension nouvelle, l'interculturalité. Comment exploiter les images de CNN sur des sujets aussi complexes que la Guerre du Golfe ou l'intervention « Restore Hope » en Somalie, ou encore, en Haïti, « Restore Democracy »? Bernard Miège (1989) affirme ainsi que :

l'avancée des industries de la communication s'accompagne d'une déstructuration/ restructuration de la fonction éducative et de l'appareil éducatif (le processus est déjà engagé pour l'enseignement des langues étrangères, rendant difficile la détermination de la fonction de médiation que va jouer l'école). 
d'admettre que tous les modèles de situations professionnelles stéréotypés, linéaires et réducteurs ne sont plus du tout adaptés à ces systèmes de relations de plus en plus ouverts, de plus en plus complexes et de plus en plus interactifs. Mettre en avant l'importance des facteurs économiques et, de ce fait, de certains points de repère en termes de potentialités, de contraintes, d'enjeux et d'objectifs comme le firent les travaux de Fast conduit le système éducatif à faire émerger un nouveau «modèle» de citoyenneté.

\section{Le retour de l'acteur}

Alain Touraine (1984) parle du retour de l'acteur en un sens autre que théâtral. Nous avons envie de dire qu'il y a urgence à articuler à nouveau «l'outil formation » autour d'un projet global, comme celui du Siècle des Lumières. L'avenir n'est pas tout écrit par d'autres. Les citoyens du monde, si les circonstances le permettent, l'écriront à leur façon, pour les grands enjeux de la démographie, de l'environnement, de l'utilisation des ressources, etc. Pourtant, l'image de l'étudiant français que nous donne D. Lapeyronnie 
(1992) à partir d'une enquête menée dans nos universités est inquiétante et risque d'être un frein à la mise en œuvre de notre projet :

L'étudiant est donc alternativement un individu (défini par son être social) et une personne (défini en soi, par sa personnalité autonome) sans que s'établisse un lien entre ces deux pôles. (1992: 94)

Nous nous efforçons, à notre modeste niveau, par une pédagogie adaptée, de réconcilier ces deux composantes en montrant comment l'apprenant passe du moi au nous, dans une démarche harmonieuse qui le conduit aussi du local au global, du travail individuel au partage avec le groupe.

Nous ne nions pas que ce projet d'interculturalité soit difficile à construire et ambitieux. Nous avons montré les fractures et les déchirements qui s'instaurent autour des notions de progrès, de travail, de révolution technique. Malgré tout, nous pensons que l'ESP est le lieu idéal pour placer la communication et la culture des autres au centre des apprentissages. Alors que l'ESP souffrait parfois autrefois d'être marginalisé en prestataire de service pour techniciens et ingénieurs, cette discipline représente aujourd'hui un atout incontournable pour l'explicitation des changements en cours, pour la transformation du paysage culturel mondial et pour la définition d'une citoyenneté planétaire.

\section{BIBLIOGRAPHIE}

D'Espagnat, B. 1990. Penser la science. Paris : Gauthier-Villars.

Fanning, P. 1993. « Broadening the ESP Umbrella ». English for Specific Purposes 12/2, 159-170.

Johnson, K. and K. Morrow. 1981. Communication in the class-room. Londres : Longman.

Lapeyronnie, D et J.-L. Marie. 1992. Campus Blues. Paris : Seuil.

Miège, B. 1989. La société conquise par la communication. Grenoble : Presses Universitaires de Grenoble.

Perrin, M. 1982. « Cohérence dans l'éclectisme : l'andragogie des langues pour la décennie à venir ». Formation France janvier, 12-15.

Touraine, A. 1984. Le retour de l'acteur. Paris : Fayard.

Witteck, F. 1989. « Cultural and linguistic diversity in the education system of the European Community ». Invited paper at the 1st International Conference of OIUNT, Varsovie, Pologne. The European Journal of Intercultural Studies, An International Forum for Intercultural Education 4/2, 7-17.

\section{NOTES}

1. Voir les analyses des résultats du programme FAST, Bruxelles, Janvier 1984, par Riccardo Petrella et Olivier Ruyssen et des documents portant sur des thèmes spécifiques, le document 
FAST FS4 sur Service, Employment: Trends and prospects et le document FAST FS4 FOP 34 sur Innovation et emplois nouveaux.

2. Language and intercultural training, revue publiée par Language Training Services à Bath (Angleterre), propose une approche pragmatique à l'intention des entreprises.

\section{RÉSUMÉS}

$\mathrm{Au}$ moment où chacun reconnaît la complexité de l'évolution de nos sociétés et l'interdépendance entre les nations, le moment semble venu de réfléchir sur le rôle de l'apprentissage des langues et en particulier sur le rôle de la langue 2 dans la formation des techniciens et des ingénieurs. Notre réflexion sur l'interculturalité participe de cette nécessaire réévaluation de nos pratiques et de nos objectifs.

At a time when international economic factors are changing rapidly all over the world, the promotion of ESP should provide learners with an intercultural education. ESP adopting a comprehensive interdisciplinary approach will encourage the mobility of technicians and engineers and provide recognition of and respect for cultural differences.

\section{INDEX}

Mots-clés : anglais de spécialité, approche, formation, interculturel

Keywords : approach, ESP, intercultural, training

\section{AUTEUR}

\section{EVELYNE BROUZENG}

Evelyne Brouzeng enseigne au Département de GEII, IUTA, Université de Bordeaux 1. evelyne.brouzeng@wanadoo.fr 